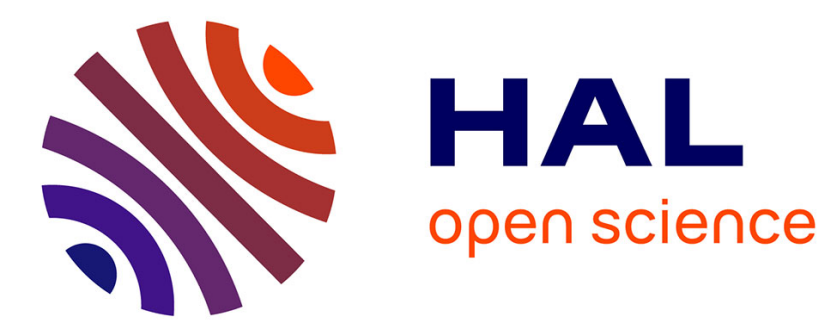

\title{
A stochastic multiple gradient descent algorithm
}

Quentin Mercier, Fabrice Poirion, Jean-Antoine Desideri

\section{To cite this version:}

Quentin Mercier, Fabrice Poirion, Jean-Antoine Desideri. A stochastic multiple gradient descent algorithm. European Journal of Operational Research, 2018, pp.10. 10.1016/j.ejor.2018.05.064 . hal-01833165

\section{HAL Id: hal-01833165 \\ https://hal.science/hal-01833165}

Submitted on 9 Jul 2018

HAL is a multi-disciplinary open access archive for the deposit and dissemination of scientific research documents, whether they are published or not. The documents may come from teaching and research institutions in France or abroad, or from public or private research centers.
L'archive ouverte pluridisciplinaire HAL, est destinée au dépôt et à la diffusion de documents scientifiques de niveau recherche, publiés ou non, émanant des établissements d'enseignement et de recherche français ou étrangers, des laboratoires publics ou privés. 


\section{Accepted Manuscript}

A Stochastic Multiple Gradient Descent Algorithm

Quentin Mercier, Fabrice Poirion, Jean-Antoine Désidéri

PII:

S0377-2217(18)30483-1

DOI:

10.1016/j.ejor.2018.05.064

Reference:

EOR 15177

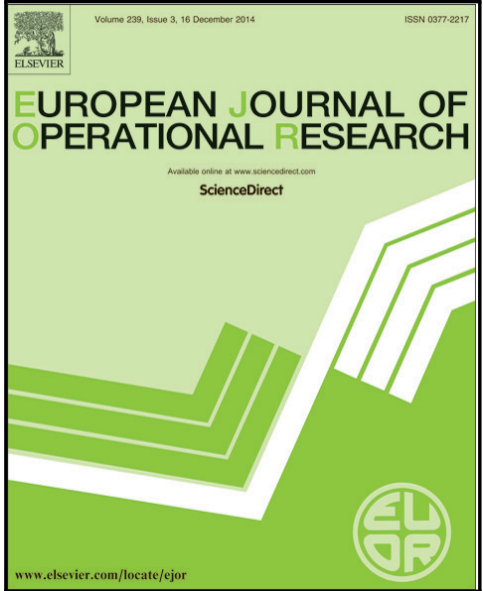

To appear in:

European Journal of Operational Research

Received date: 2 March 2017

Revised date: $\quad 6$ April 2018

Accepted date: $\quad 23$ May 2018

Please cite this article as: Quentin Mercier, Fabrice Poirion, Jean-Antoine Désidéri, A Stochastic Multiple Gradient Descent Algorithm, European Journal of Operational Research (2018), doi: 10.1016/j.ejor.2018.05.064

This is a PDF file of an unedited manuscript that has been accepted for publication. As a service to our customers we are providing this early version of the manuscript. The manuscript will undergo copyediting, typesetting, and review of the resulting proof before it is published in its final form. Please note that during the production process errors may be discovered which could affect the content, and all legal disclaimers that apply to the journal pertain. 


\section{Highlights}

- A new gradient-based algorithm for stochastic multiobjective optimization problem

- Mean-square and almost-sure convergence of the algorithm proven

- Algorithm tested on a variety of benchmark tests

- Performance compared to two optimization algorithms coupled with a Monte Carlo estimator

- Algorithm computationally efficient

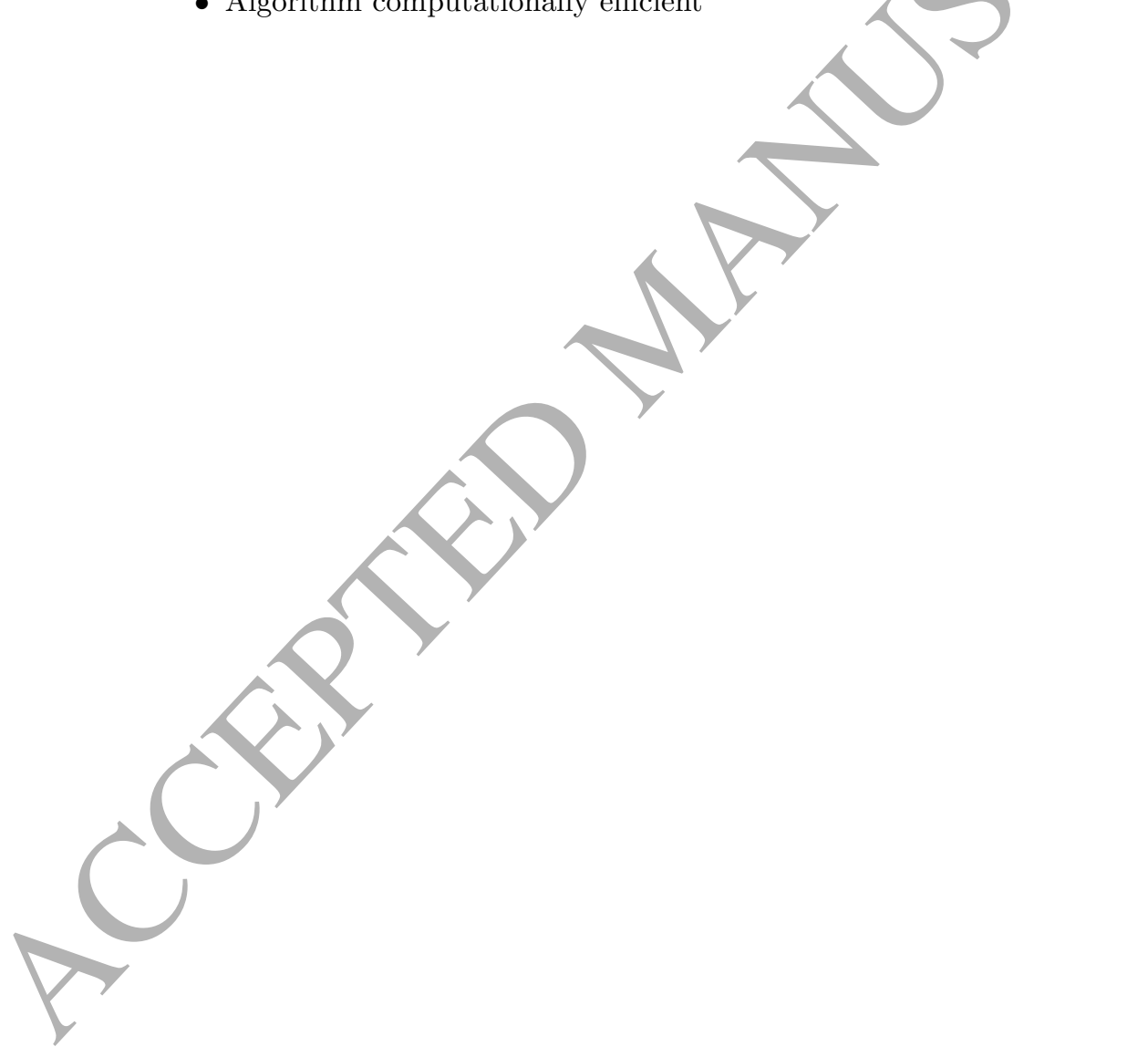




\title{
A Stochastic Multiple Gradient Descent Algorithm
}

\author{
${\text { Quentin } \text { Mercier }^{\mathrm{a}, *}, \text { Fabrice Poirion }}^{\mathrm{a}}$, Jean-Antoine Désidéri ${ }^{\mathrm{b}}$ \\ ${ }^{a}$ Onera the French Aerospace Lab, 29 avenue de la Division Leclerc, 92320 Chtillon, France \\ ${ }^{b}$ Inria, 2004 Route des Lucioles, 06902 Valbonne, France
}

\section{Abstract}

In this article, we propose a new method for multiobjective optimization problems in which the objective functions are expressed as expectations of random functions. The present method is based on an extension of the classical stochastic gradient algorithm and a deterministic multiobjective algorithm, the Multiple Gradient Descent Algorithm (MGDA). In MGDA a descent direction common to all specified objective functions is identified through a result of convex geometry. The use of this common descent vector and the Pareto stationarity definition into the stochastic gradient algorithm makes the algorithm able to solve multiobjective problems. The mean square and almost sure convergence of this new algorithm are proven considering the classical stochastic gradient algorithm hypothesis. The algorithm efficiency is illustrated on a set of benchmarks with dixerse complexity and assessed in comparison with two classical algorithms (NSGA-II, DMS) coupled with a Monte Carlo expectation estimator.

Keywords: Multiple objective programming, Multiobjective stochastic optimization, Stochastic gradient algorithm, Multiple gradient descent algorithm, Common descent vector

* Corresponding author

Email addresses: quentin.mercier@onera.fr (Quentin Mercier), poirion@onera.fr (Fabrice Poirion), jean-antoine.desideri@inria.fr (Jean-Antoine Désidéri) 


\section{Introduction}

Manufacturers are ever looking for designing products with better performance, higher reliability at lower cost and risk. One way to address these antagonistic objectives is to use multiobjective optimization approaches. But 5 real world problems are rarely described through a collection of fixed parameters and uncertainty has to be taken into account, may it appear in the system description itself or in the environment and operational conditions. Indeed the system behavior can be very sensitive to modifications in some parameters $[1,30,33]$. This is why uncertainty has to be introduced in the design 10 process from the start. Optimization under uncertainty has known important advances since the second-half of the 20 th century $[4,9,28]$ and various approaches have been proposed including robust optimization, which encompasses today a rather large field of robustness concepts such as the "worst case" or the "mean and variance" concepts [26], and stochastic optimization where uncer15 tain parameters are modeled through random variables with a given distribution and where the probabilistic information is directly introduced in the numerical approaches. In that context the uncertain multiobjective problems are written in terms of the expectation of each objective. In our paper we shall focus on this last interpretation of the optimization problem. Considering single objec-

20 tive stochastic optimization problems, a large variety of numerical approaches $[36,37]$ can be found in the literature. Two main distinct approaches exist, one based on stochastic approximations such as the Robbins Monro algorithm and the various stochastic gradient approaches [21, 22, 35], the second one based on scenario approaches $[32,39]$, the latter being more frequently applied for chance constrained problems.

Regarding stochastic multiobjective optimization the literature is less prolific: the various approaches proposed are based on classical deterministic algorithm such as genetic algorithms coupled with a robust formulation where the random quantities appearing (such as the mean values or standard deviations) 30 are either obtained analytically for simple objectives [7, 11, 25] or estimated 
using a sample averaging approach using scenarios $[5,18,24,29,31,40]$. In this paper, we propose a new algorithm for constructing the set of Pareto stationary points of a multiobjective optimization problem written in terms of the mean objective functions. The method is based on the use of the $M G D A$ algorithm $35[12,14]$ and more precisely on the existence of a common descent vector analogous to the steepest descent vector of [23], together with a stochastic gradient algorithm. Convergences of this new algorithm will be proved and several i1lustrations given. The paper is organized as follows. In section 2 the Multiple Gradient Descent Algorithm ( $M G D A)$ is recalled. In section 3, after introducing some probabilistic notations and results which will be used for the convergence proofs, we introduce the problem under consideration and introduce the Stochastic Multiple Gradient Descent Algorithm ( $S M G D A)$. Then we shall prove two types of convergence. In section 4 illustrations of the $S M G D A$ algorithm will be given and compared to the classical Sample Average Approximation (SAA) 45 approach [39].

\section{Multiple Gradient Descent Algorithm ( $M G D A)$}

The Multiple Gradient Descent Algorithm (MGDA) was originally introduced in [13] and [12] to solve general multiobjective optimization problems involving differentiable cost functions. Variants were proposed in [14], but more 50 recently the algorithm was slightly revised in [15] to apply to cases where the number $m$ of objective functions exceeds the dimension $n$ of the working design space

Recently, the revised version of $M G D A$ was applied in a deterministic setto a time periodic problem governed by the time dependent compressible Navier-Stokes equations [17]. There, six parameters defining pulsating jets on a flat plate have been optimized to reduce drag over a time period. The multiple gradients were the realizations at 800 time steps of the gradient of drag with respect to the six parameters. By $M G D A$, drag was reduced at every time steps of the period. Presently, following [17], we summarize the method used 
60 to construct the descent direction from a set made of multiple gradients, using slightly different notations being necessary in the subsequent stochastic framework. For the sake of clarity, the calculation of the common descent vector is only presented in the linearly independent gradients case where the number of objectives $m$ is supposed inferior to the dimension of the design space $n$.

\section{2.1. Multiobjective problem statement}

Let $m$ and $n$ be two arbitrary integers and consider the multiobjective optimization problem consisting in minimizing $m$ differentiable objective functions $\left\{f_{j}(\mathbf{x})\right\}$ in some open admissible domain $\mathcal{D}_{a} \subseteq \mathbb{R}^{n}\left(j=1, \ldots, m ; f_{j} \in C^{1}\left(\mathcal{D}_{a}\right)\right)$. Given a starting point $\mathbf{x}_{0} \in \mathcal{D}_{a}$ and a vector $\mathbf{d} \in \mathbb{R}^{n}$, one forms the directional derivatives

$$
f_{j}^{\prime}=\left[\nabla_{\mathbf{x}} f_{j}\left(\mathbf{x}_{0}\right)\right]^{t} \mathbf{d}
$$

where $\nabla_{\mathbf{x}}$ is the symbol for the gradient w.r.t. $\mathbf{x}$ and the superscript ${ }^{t}$ stands for transposition. One seeks for a vector $\mathbf{d}$ such that the scalar product of any objective gradient $\nabla_{x} f_{j}\left(\mathbf{x}_{0}\right)$ with the vector $\mathbf{d}$ remains strictly positive

$$
f_{j}^{\prime}>0
$$

If such a vector $\mathbf{d}$ exists, the direction of the vector $(-\mathbf{d})$ is said to be a local descent direction common to all objective functions. Then evidently, infinitely many other such directions also exist, and our algorithm permits to identify at least one.

2.2. Convex hull, two lemmas and basic MGDA

We recall the following :

Definition 1. The convex hull of a family of $m$ vectors $\left\{\mathbf{u}_{j}\right\}(j=1, \ldots, m$; $\left.\mathbf{u}_{j} \in \mathbb{R}^{n}\right)$, is the set of all their convex combinations

$$
\overline{\mathcal{U}}=\left\{\mathbf{u} \in \mathbb{R}^{n} \text { such that } \mathbf{u}=\sum_{j=1}^{m} \alpha_{j} \mathbf{u}_{j} ; \alpha_{j} \in \mathbb{R}^{+}(\forall j) ; \sum_{j=1}^{m} \alpha_{j}=1\right\} .
$$


Evidently, given the $m$ vectors $\left\{\mathbf{u}_{j}\right\}$ in $\mathbb{R}^{n}$, the convex hull $\overline{\mathcal{U}}$ is a convex, closed and bounded subset of the finite-dimensional subspace spanned by these vectors. Its image in the affine space $\mathbb{R}^{n}$ in which vectors are associated with 75 representatives of same origin $\mathrm{O}$, is a convex polytope with at most $m$ vertices. Then, we have :

Lemma 1. Given an $n \times n$ real-symmetric positive-definite matrix $\mathbf{A}_{n}$, the associated scalar product

$$
(\mathbf{u}, \mathbf{v})=\mathbf{u}^{t} \mathbf{A}_{n} \mathbf{v} \quad\left(\mathbf{u}, \mathbf{v} \in \mathbb{R}^{n}\right),
$$

and Euclidean norm

$$
\|\mathbf{u}\|=\sqrt{\mathbf{u}^{t} \mathbf{A}_{n} \mathbf{u}}
$$

the convex hull $\overline{\mathcal{U}}$ admits a unique element $\xi^{\star}$ of minimum norm.

Proof. - Existence $: \overline{\mathcal{U}}$ is compact and \|.\| is a continuous function.

- Uniqueness : suppose that $\xi_{1}$ and $\xi_{2}$ are two realizations of the minimum $\mu=\arg \min _{\mathbf{u} \in \overline{\mathcal{U}}}\|\mathbf{u}\|$ so that $\mu=\left\|\xi_{1}\right\|=\left\|\xi_{2}\right\|$ and let

$$
\xi_{s}=\frac{1}{2}\left(\xi_{2}+\xi_{1}\right), \quad \xi_{d}=\frac{1}{2}\left(\xi_{2}-\xi_{1}\right),
$$

so that:

$$
\left(\xi_{s}, \xi_{d}\right)=\frac{1}{4}\left(\xi_{2}+\xi_{1}, \xi_{2}-\xi_{1}\right)=\frac{1}{4}\left(\left\|\xi_{2}\right\|^{2}-\left\|\xi_{1}\right\|^{2}\right)=0 .
$$

Hence $\xi_{s} \perp \xi_{d}$, and since $\xi_{s} \in \overline{\mathcal{U}},\left\|\xi_{s}\right\| \geq \mu$, and

$$
\mu^{2}=\left\|\xi_{2}\right\|^{2}=\left\|\xi_{s}+\xi_{d}\right\|^{2}=\left\|\xi_{s}\right\|^{2}+\left\|\xi_{d}\right\|^{2} \geq \mu^{2}+\left\|\xi_{d}\right\|^{2} \Longrightarrow \xi_{d}=0
$$

$$
\left(\mathbf{u}, \xi^{\star}\right) \geq\left\|\xi^{\star}\right\|^{2}
$$


Proof. Let $\mathbf{u} \in \overline{\mathcal{U}}$, arbitrary. Let $r=\mathbf{u}-\xi^{\star}$; by convexity of $\overline{\mathcal{U}}$, any convex combination of $\xi^{\star}$ and $\mathbf{u}$ is an element of $\overline{\mathcal{U}}$

$$
(1-\epsilon) \xi^{\star}+\epsilon \mathbf{u}=\xi^{\star}+\epsilon r \in \overline{\mathcal{U}}, \epsilon \in[0,1] .
$$

By definition of $\xi^{\star},\left\|\xi^{\star}+\epsilon r\right\| \geq\left\|\xi^{\star}\right\|$, that is

$$
\left(\xi^{\star}+\epsilon r, \xi^{\star}+\epsilon r\right)-\left(\xi^{\star}, \xi^{\star}\right)=2 \epsilon\left(\xi^{\star}, r\right)+\epsilon^{2}\|r\|^{2} \geq 0
$$

and this requires that the coefficient $\left(\xi^{\star}, r\right)$ of $\epsilon$ be non-negative.

Then consider the set $\left\{\mathbf{u}_{j}\right\}_{j \in \llbracket 1, m \rrbracket}$ where each element is the gradient of the objective function $f_{j}$ at point $\mathbf{x}_{0}$

$$
\mathbf{u}_{j}=\nabla_{\mathbf{x}} f_{j}\left(\mathbf{x}_{0}\right)
$$

If the vector $\xi^{\star}$ defined in Lemma 1 is nonzero, the vector

$$
\mathbf{d}=\mathbf{A}_{n}
$$

is also nonzero, and is a solution to the problem stated in (1)-(2) since by virtue of Lemma 2

$$
\left(\mathbf{u}_{j}, \xi^{\star}\right)=\mathbf{u}_{j}^{t} \mathbf{A}_{n} \xi^{\star}=\mathbf{u}_{j}^{t} \mathbf{d} \geq\left\|\xi^{\star}\right\|^{2}>0 .
$$

The situation in which $\xi^{\star}=0$, or equivalently, when there exists a set $\alpha=\left\{\alpha_{j}\right\}$ of $m$ positive real numbers such that

$$
\sum_{j=1}^{m} \alpha_{j} \nabla f_{j}\left(\mathbf{x}_{0}\right)=0 \text { and } \sum_{j=1}^{m} \alpha_{j}=1
$$

80 is said to be one of "Pareto stationarity". The relationship between Pareto optimality and Pareto stationarity was made precise by the following [14]-[15]

Theorem 1. If the objective functions are differentiable and convex in some open ball $\mathcal{B} \subseteq \mathcal{D}_{a}$ about $\mathbf{x}_{0}$, and if $\mathbf{x}_{0}$ is Pareto optimal, then the Pareto stationarity condition is satisfied at $\mathbf{x}_{0}$.

Proof. Without loss of generality, suppose that $f_{j}\left(\mathbf{x}_{0}\right)=0$ for $j \in \llbracket 1, m \rrbracket$. Since, by hypothesis, $\mathbf{x}_{0}$ is Pareto optimal, a single, arbitrary objective function cannot 
be improved (here diminished below 0) under the constraint of no-degradation of the others. In particular, $\mathbf{x}_{0}$ solves the problem

$$
\min _{\mathbf{x}} f_{m}(\mathbf{x}) / \text { subject to }: f_{j}(\mathbf{x}) \leq 0, j \in \llbracket 1, m-1 \rrbracket .
$$

Let $\overline{\mathcal{U}}_{m-1}$ be the convex hull of the $m-1$ gradients $\left\{\mathbf{u}_{1}, \mathbf{u}_{2}, \ldots, \mathbf{u}_{m-1}\right\}$ and

$$
\xi_{m-1}^{\star}=\underset{\mathbf{u} \in \overline{\mathcal{U}}_{m-1}}{\operatorname{argmin}}\|\mathbf{u}\| .
$$

Existence, uniqueness and following property of this element have already been established (Lemmas 2.1 and 2.2)

$$
\left(\mathbf{u}_{j}, \xi_{m-1}^{\star}\right) \geq\left\|\xi_{m-1}^{\star}\right\|^{2}, j \in \llbracket 1, m_{\text {工 }} 1 \rrbracket .
$$

${ }_{85}$ Two situations are then possible :

1. Either $\xi_{m-1}^{\star}=0$, and the Pareto stationarity condition is satisfied at $\mathbf{x}=\mathbf{x}_{0}$ with $\alpha_{m}=0$.

2. Or $\xi_{m-1}^{\star} \neq 0$. Then let $\phi_{j}(\epsilon)=f_{j}\left(\mathbf{x}_{0}>\epsilon \xi_{m-1}^{\star}\right)(j=1, \ldots, m-1)$ so that $\phi_{j}(0)=0$ and $\phi_{j}^{\prime}(0)=-\left(\mathbf{u}_{j}, \xi_{m-1}^{\star}\right) \leq-\left\|\xi_{m-1}^{\star}\right\|^{2}<0$, and for sufficiently-small $\epsilon$

$$
\phi_{j}(\epsilon)=f_{j}\left(\mathbf{x}_{0}-\epsilon \xi_{m-1}^{\star}\right)<0, j \in \llbracket 1, m-1 \rrbracket .
$$

This result confirms that for the constrained minimization problem (11), Slater's constraint-qualification criterion is satisfied, and optimality requires the satisfaction of the Karush-Kuhn-Tucker (KKT) condition [6], that is, the Lagrangian

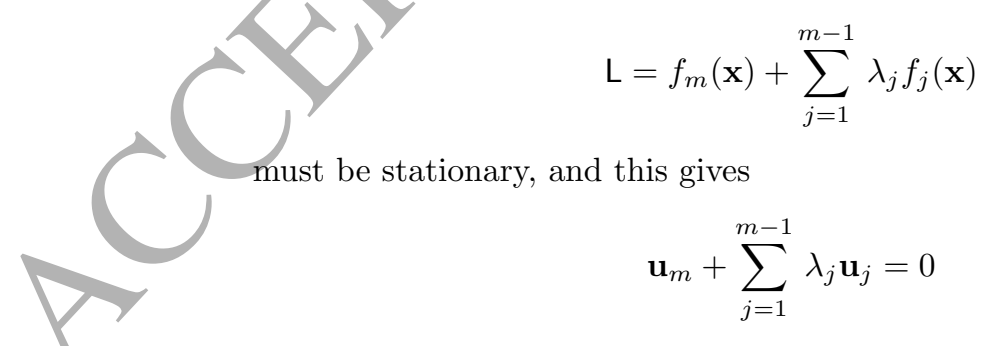

in which $\lambda_{j}>0$ for $j \in \llbracket 1, m-1 \rrbracket$ by saturation of the constraints $\left(f_{j}\left(\mathbf{x}_{0}\right)=0\right)$ and sign convention. Finally, $\Lambda=1+\sum_{j=1}^{m-1} \lambda_{j}>1$. Thus 90 by dividing the above equation by $\Lambda \neq 0$, the result is achieved. 
Note that this proof is valid for all $m$ and $n$, in particular in situations in which $m \geq n$, encountered in particular in multi-point optimization when the number of points is larger than the number of variables, as well as $m<n$ more 95 typical of multidisciplinary optimization.

Hence, the Pareto stationarity condition generalizes to the multiobjective context, the classical stationarity condition expressing that an unconstrained differentiable function is extremal.

We now return to the non-trivial case of a point $\mathbf{x}_{0}$ that is not Pareto stationary and we suppose that the vectors $\xi^{\star}$ and $\mathbf{d}\left(\xi^{\star} \neq 0 ; \mathbf{d} \neq 0\right)$ have been identified (see next subsection). Then we define $M G D A$ as the iteration which transforms $\mathbf{x}_{0}$ in

$$
\mathbf{x}_{1}=\mathbf{x}_{0}-\rho \mathbf{d}
$$

where $\rho>0$ is some appropriate step size. In many cases in engineering sciences, the step size can be adjusted after an analysis of the the physical scales involved is made [17]. PDE-constrainted optimization is our ultimate goal, and realistically, the number of cost function evaluations per optimization iteration should remain small. Additionally, in steady problems, a constant step size is often adequate although not optimal. This is our choice here. Nevertheless, in case where step size adaptation is really beneficial, it may be realized by constraint violation limitation, and/or accurate, or coarse one dimensional optimization in the direction of search; see for exemple [16].

Thus MGDA is an extension to the multiobjective context of the classical steepest descent method, in which the direction of search is taken to be the vector d defined above. At convergence, the limiting point is Pareto stationary. We now examine how can the vector $\mathbf{d}$ be computed in practice.

\subsection{QP formulation and hierarchical Gram-Schmidt orthogonalization}

By letting

$$
\xi^{\star}=\sum_{j=1}^{m} \alpha_{j} \mathbf{u}_{j}=\mathbf{U} \alpha
$$


where $\mathbf{u}_{j}=\nabla_{\mathbf{x}} f_{j}\left(\mathbf{x}_{0}\right)$, $\mathbf{U}$ is the $n \times m$ matrix whose $j$ th column contains the $n$ components of vector $\mathbf{u}_{j}$, the identification of vector $\xi^{\star}$ can be made by solving the following Quadratic Programming (QP) problem for the unknown vector of coefficients $\alpha=\left\{\alpha_{j}\right\}$

$$
\xi^{\star}=\arg \min _{\alpha \in \mathbb{R}^{m}} \frac{1}{2} \alpha^{t} \mathbf{H} \alpha
$$

subject to

$$
\alpha_{j} \geq 0, j \in \llbracket 1, m \rrbracket, \quad \sum_{j=1}^{m} \alpha_{j}=1,
$$

where $\mathbf{H}=\mathbf{U}^{t} \mathbf{A}_{n} \mathbf{U}$. Note that if vector $\xi^{\star}$ is unique, vector $\alpha$ may not be.

However, if the family of gradients is linearly-independent, which requires in particular that $m \leq n$, it is possible to choose the scalar product, through the definition of matrix $\mathbf{A}_{n}$, in such a way that the given gradients are 2 by 2 orthogonal, and of norm 1 . Then the QP-formulation admits the trivial solution

$$
\xi^{\star}=\frac{1}{m} \sum_{j=1}^{m} \mathbf{u}_{j}
$$

To characterize matrix $\mathbf{A}_{n}$, first apply a Gram-Schmidt orthogonalization process to the vectors $\left\{\mathbf{u}_{j}\right\}$ and get a new family of vectors, $\left\{\mathbf{v}_{j}\right\}, 2$ by 2 orthogonal with respect to the standard Euclidean scalar product $\left(\mathbf{v}_{j}^{t} \mathbf{v}_{k}=0, j \neq k\right)$; define the following diagonal matrix

$$
\Delta=\operatorname{Diag}\left(\mathbf{v}_{j}^{t} \mathbf{v}_{j}\right)
$$

Then:

$$
\mathbf{A}_{n}=\mathbf{W}^{t} \mathbf{W}+(\mathbf{I}-\Pi)^{2}, \quad \mathbf{W}=\left(\mathbf{U}^{t} \mathbf{U}\right)^{-1} \mathbf{U}^{t} \mathbf{V} \Delta^{-1} \mathbf{V}^{t}
$$

where $\Pi=\mathbf{V} \Delta^{-1} \mathbf{V}^{t}$ is the projection matrix onto subspace spanned by the gradients [15].

Once matrix $\mathbf{A}_{n}$ is defined, the descent direction (-d) is given by (8), and a descent step can be performed by $M G D A,(17)$.

Remark 1. In the case of a linearly-dependent family of gradients, only a subfamily of $\left\{\mathbf{u}_{j}\right\}$ is used in the Gram-Schmidt process. The elements of this 
subfamily are selected one-by-one according to a specific hierarchical principle which tends to make the cone associated with the hull of the subfamily as large as possible. A new family of same rank and made of vectors two-by-two orthogonal is thus constructed, $\left\{\mathbf{v}_{j}\right\}$. Then, one usually resorts to solving the QP-problem but reformulated in this basis which permits a very stable numerical treatment $[15,17]$. The case of exception is when Pareto stationarity is detected; then, the algorithm is terminated. However, in any case, this methodological enhancement is not necessary here since the number $m$ of objective functions is less than the dimension $n$ of the admissible working space, and the gradients are assumed to be linearly independent.

\section{The Stochastic Multiple Gradient Descent Algorithm (SMGDA)}

Using results given in the previous section, we are going to extend the $M G D A$ algorithm to the stochastic context using a stochastic gradient like algorithm. Classical probabilistic results are recalled in the first subsection. Then the problem formulation and the SMGDA algorithm are described. In the last subsection two convergence results aré given.

\subsection{Probabilistic prerequisites}

Let $(\Omega, \mathcal{A}, \mathbb{P})$ be an abstract probabilistic space, and $W: \Omega \rightarrow \mathbb{R}^{d}, \omega \mapsto W(\omega)$ a given random vector. We denote $\mu$ the distribution of the random variable $W$ and $\mathcal{W}$ its image space $W(\Omega) \subset \mathbb{R}^{d}$. Let $W_{1}, \ldots, W_{p}, \ldots$ be independent copies ${ }_{140}$ of the random variable $W$ which will be used to generate independent random samples with distribution $\mu$. We denote $\mathcal{F}_{k}=\sigma\left(W_{1}, \ldots, W_{k}\right)$ the $\sigma$-algebra generated by the $k$ first random variables $W_{i}$. Since $\mathcal{F}_{k-1} \subset \mathcal{F}_{k}$ the sequence $\left\{\mathcal{F}_{k}\right\}_{k \geq 1}$ is a filtration denoted $\mathcal{F}$.

Definition 2. A sequence $\left(X_{n}\right)$ of integrable random variables is a supermartingale relatively to the filtration $\mathcal{F}$ if $X_{n}$ is $\mathcal{F}_{n}$ measurable and if and only if

$$
\mathbb{E}\left(X_{n+1} \mid \mathcal{F}_{n}\right) \leq X_{n}
$$


almost surely (a.s.) where $\mathbb{E}\left(X_{n+1} \mid \mathcal{F}_{n}\right)$ denotes the conditional expectation of ${ }_{145}$ the random variable $X_{n+1}$ respectively to the $\sigma$-algebra $\mathcal{F}_{n}$.

The two following classical results will be used in the convergence proof:

Lemma 3. Let $\mathcal{B} \subset \mathcal{A}$ be two $\sigma$-algebras from probability space $(\Omega, \mathcal{A}, \mathbb{P})$ and $X$ and $Y$ be two independent random variables such that $X$ is independent of $\mathcal{B}$ and $Y$ is $\mathcal{B}$-measurable. We consider $f$, a measurable bounded function that takes its values in $\mathbb{R}$. Then

$$
\left\{\begin{array}{l}
\mathbb{E}[f(X, Y) \mid \mathcal{B}]=\varphi(Y) \\
\varphi(y)=\mathbb{E}[f(X, y)]
\end{array}\right.
$$

Theorem 2. Let $\left(X_{k}\right)_{k \in \mathbb{N}}$ be a positive supermartingale. Then there exists a random variable $X_{\infty}$ such that $X_{k}$ converge toward $X_{\infty}$ almost surely

\subsection{Problem statement}

$$
\mathbb{P}\left(\lim _{k \rightarrow \infty} X_{k}=X_{\infty}\right)=1 \text {. }
$$

Throughout the paper the standard inner product on $\mathbb{R}^{n}$ will be used and denoted $\langle\cdot, \cdot\rangle$, the norm being denoted $\|\cdot\|$.

Consider $m$ functions $f_{j}: \mathbb{R}^{n} \times \mathcal{W} \rightarrow \mathbb{R}, j=1, \ldots, m$. The problem addressed in this paper is to solve the mean multiobjective optimization problem written

$$
\min _{\mathbf{x} \in \mathbb{R}^{n}}\left\{\mathbb{E}\left[f_{1}(\mathbf{x}, W), \mathbb{E}\left[f_{2}(\mathbf{x}, W)\right], \ldots, \mathbb{E}\left[f_{m}(\mathbf{x}, W)\right]\right\}\right.
$$

More precisely we want to construct a set of points that belongs to the associated Pareto set. As it is written, problem (24) is a deterministic problem but in general the objective function expectations are not known. A classical approach is to replace each expectancy by an estimator built using independent samples $w_{k}$ of the random variable $W[5,24]$. As for stochastic gradient algorithms, the algorithm we propose does not need to calculate the mean objective functions and is only based on the values of the stochastic functions gradients. The classical stochastic gradient algorithm is based on a descent direction given by the objective function gradient. Here the descent vector which will be used is 
the common descent vector constructed in the previous section for the $M G D A$ algorithm. In the stochastic context this common descent vector is random and defined by the random convex combination

$$
\xi^{\star}(\mathbf{x}, W)=\sum_{j=1}^{m} \alpha_{j}(\mathbf{x}, W) \nabla f_{j}(\mathbf{x}, W) \text { a.s., } \mathbf{x} \in \mathbb{R}^{n}
$$

with

$$
\sum_{j} \alpha_{j}(\mathbf{x}, W)=1 \text { a.s. }
$$

by construction.

The flow chart of SMGDA ( Stochastic Multiple Gradient Descent Algorithm) algorithm is described below.

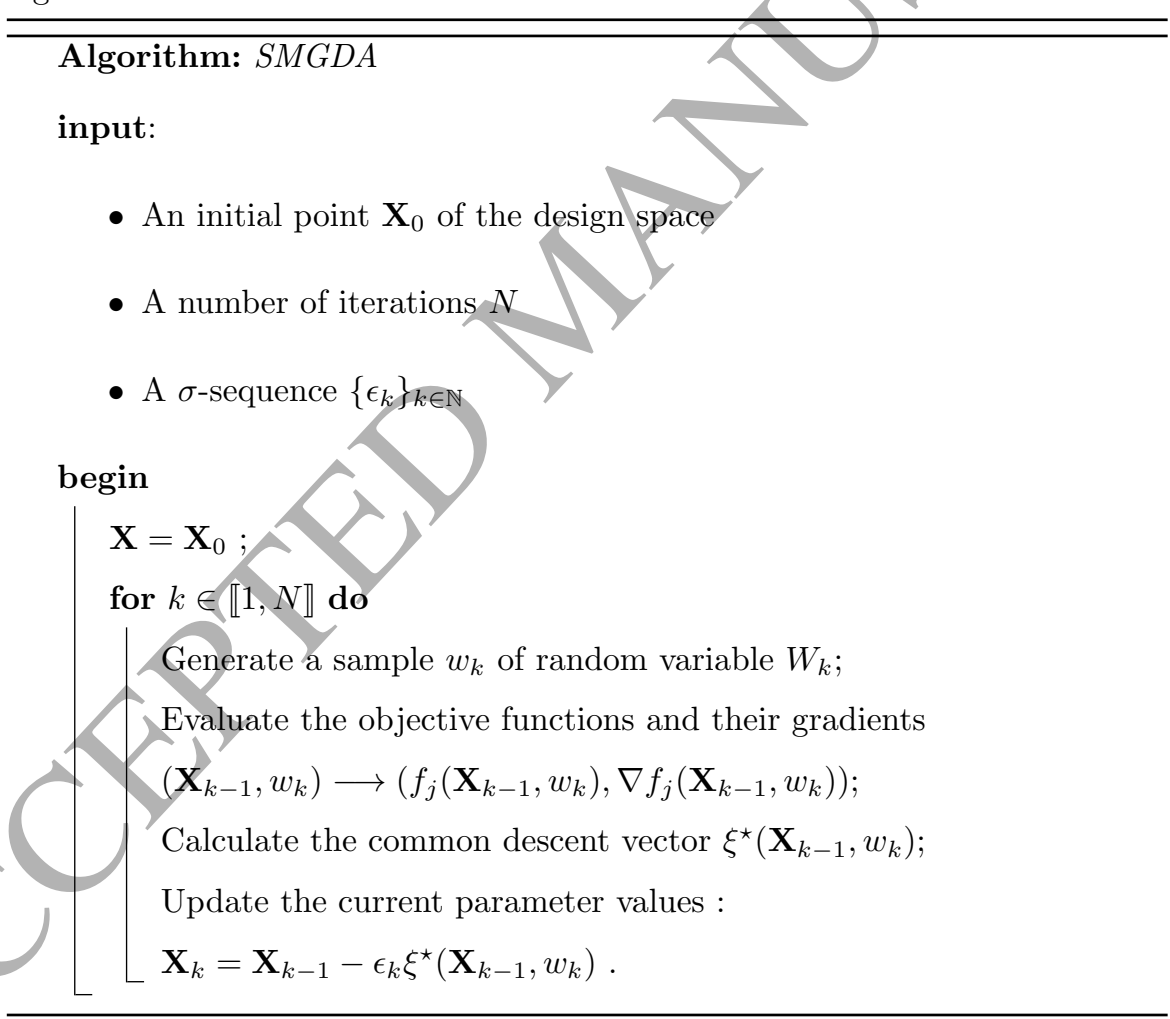

Remark 2. Two parameters require user adjustment: the number of iterations 155 for the stochastic algorithm, and the $\sigma$-sequence that define the step size. 
Remark 3. No stopping criterion is proposed as it is the case for most stochastic algorithms since there exists no efficient ones.

More generally we shall consider the random sequence $\left(\mathbf{X}_{k}\right)$ defined by the recurrence relation

$$
\mathbf{X}_{k}=\mathbf{X}_{k-1}-\epsilon_{k} \xi^{\star}\left(\mathbf{X}_{k-1}, W_{k}\right)
$$

\subsection{Convergence proofs}

Two types of convergences will be proved in this section, the first one being a mean square convergence in the Hilbert space $L^{2}(\Omega)$, the second one being an almost sure point-wise convergence. The two proofs are extensions of the stochastic gradient convergence proofs and are based on classical assumptions which can be found for instance in [19, 34].

The notation $\mathcal{P}_{D}^{\star}$ (resp. $\mathcal{P}_{O}^{\star}$ ) will denote the Pareto solution set (resp. the Pareto front). For any $\mathbf{x} \in \mathbb{R}^{n}$ the notation $\mathbf{x}^{\perp}$ will denote an element of the Pareto set which minimizes the distaníce between the point $\mathbf{x}$ and a point of the Pareto set $\mathcal{P}_{D}{ }^{\star}$

$$
\mathbf{x}^{\perp} \in \underset{\mathbf{u} \in \mathcal{P}_{D}^{\star}}{\operatorname{argmin}}\{\|\mathbf{x}-\mathbf{u}\|\} .
$$

The convergence results rely upon the following hypotheses.

${ }_{165}$ H1 Problem (24) admits a nonempty Pareto solution set $\mathcal{P}_{D}^{\star}$.

H2 The random variables $f_{j}(\mathbf{x}, W)$ are integrable for $j=1, \ldots, m$ and for all $\mathrm{x} \in \mathbb{R}^{n}$

H3 The functions $\mathbf{x} \mapsto f_{j}(\mathbf{x}, W): \mathbb{R}^{n} \rightarrow \mathbb{R}$ are convex and their derivatives exist almost surely for $j=1, \ldots, m$.

H4 The partial gradient of function $f_{j}$ with respect to $\mathbf{x}$ is almost surely uniformly bounded by a strictly positive real number $M_{j}$

$$
\left\|\nabla f_{j}(\mathbf{x}, W)\right\| \leq M_{j} \text { a.s., } \mathbf{x} \in \mathbb{R}^{n} .
$$

H5 For any objective function $f_{j}$, there exists a positive real number $c_{j}$ such that for any $\mathbf{x}$ in $\mathbb{R}^{n}$ the following relation holds

$$
f_{j}(\mathbf{x}, W)-f_{j}\left(\mathbf{x}^{\perp}, W\right) \geq \frac{c_{j}}{2}\left\|\mathbf{x}-\mathbf{x}^{\perp}\right\|^{2} \text { a.s. } ; j=1, \ldots, m .
$$


H6 The sequence $\left\{\epsilon_{k}\right\}_{k \in \mathbb{N}}$ is a $\sigma$-sequence

$$
\begin{aligned}
& \sum_{k=0}^{\infty} \epsilon_{k}=\infty \\
& \sum_{k=0}^{\infty} \epsilon_{k}^{2}<\infty .
\end{aligned}
$$

170

Some properties of the common descent vector $\xi^{\star}$ will be needed further.

Proposition 1. The norm of the common descent vector $\xi^{\star}$ is almost surely uniformly bounded by a positive real number $M_{\xi^{\star}}$

$$
\left\|\xi^{\star}(\mathbf{x}, W)\right\| \leq M_{\xi^{\star}} \text { a.s., } \mathbf{x} \in \mathbb{R}^{n} .
$$

Proof. Using the definition of the common descent vector

$$
\forall(\mathbf{x}, w) \in \mathbb{R}^{n} \times \mathcal{W} ;\left\|\xi^{\star}(\mathbf{x}, w)\right\|=\left\|\sum_{j=1}^{m} \alpha_{j}(\mathbf{x}, w) \nabla f_{j}(\mathbf{x}, w)\right\|
$$

with $0 \leq \alpha_{j}(\mathbf{x}, w) \leq 1$ and $\sum_{j} \alpha_{j}(\mathbf{x}, w)=1$, we can write

$$
\left\|\xi^{\star}(\mathbf{x}, w)\right\| \leq \sum_{j=1}^{m}\left\|\alpha_{j}(\mathbf{x}, w) \nabla f_{j}(\mathbf{x}, w)\right\| \leq \sum_{j=1}^{m}\left\|\nabla f_{j}(\mathbf{x}, w)\right\| .
$$

Under assumption $\mathrm{H} 4$

$$
\left\|\xi^{\star}(\mathbf{x}, w)\right\| \leq \sum_{j=1}^{m} M_{j}
$$

The mean common descent vector is defined as:

\section{Definition 3.}

$$
\begin{aligned}
\Xi^{\star}(\mathbf{x})=\mathbb{E}\left[\xi^{\star}(\mathbf{x}, W)\right] & =\mathbb{E}\left[\sum_{j=1}^{m} \alpha_{j}(\mathbf{x}, W) \nabla f_{j}(\mathbf{x}, W)\right] \\
& =\sum_{j=1}^{m} \mathbb{E}\left[\alpha_{j}(\mathbf{x}, W) \nabla f_{j}(\mathbf{x}, W)\right]
\end{aligned}
$$


Proposition 2. The mean common descent vector satisfies the following property for any $\mathbf{x}$ in $\mathbb{R}^{n}$

$$
\Xi^{\star}(\mathbf{x})\left(\mathbf{x}-\mathbf{x}^{\perp}\right) \geq \frac{C}{2}\left\|\mathbf{x}-\mathbf{x}^{\perp}\right\|^{2}
$$

${ }_{175}$ with $C=\min c_{j}$ and where the $c_{j}$ are defined in H5.

Proof. From hypothesis H3, we know that any objective function $f_{j}$ is almost surely convex

$$
\nabla f_{j}(\mathbf{x}, W)\left(\mathbf{x}-\mathbf{x}^{\perp}\right) \geq f_{j}(\mathbf{x}, W)-f_{j}\left(\mathbf{x}^{\perp}, W\right) \text { a.s., } \mathbf{x} \in \mathbb{R}^{n}
$$

Therefore, using assumption H5 ,

$$
\nabla f_{j}(\mathbf{x}, W)\left(\mathbf{x}-\mathbf{x}^{\perp}\right) \geq \frac{c_{j}}{2}\left\|\mathbf{x}-\mathbf{x}^{\perp}\right\|^{2} \text { a.s. }
$$

Introducing the coefficients that define $\xi^{\star}(x, W)$, we can write

$$
\sum_{j=1}^{m} \alpha_{j}(\mathbf{x}, W) \nabla f_{j}(\mathbf{x}, W)\left(\mathbf{x}-\mathbf{x}^{\perp}\right) \geq \sum_{j=1}^{m} \alpha_{j}(\mathbf{x}, W) \frac{c_{j}}{2}\left\|\mathbf{x}-\mathbf{x}^{\perp}\right\|^{2} a . s . .
$$

Let $C=\min _{j} c_{j}$, we can write

$$
\sum_{j=1}^{m} \alpha_{j}(\mathbf{x}, W) \nabla f_{j}(\mathbf{x}, W)\left(\mathbf{x}-\mathbf{x}^{\mathcal{1}}\right) \geq \frac{C}{2}\left\|\mathbf{x}-\mathbf{x}^{\perp}\right\|^{2} \sum_{j=1}^{m} \alpha_{j}(\mathbf{x}, W) a . s . .
$$

Since the $\left\{\alpha_{j}\right\}$ sum up to 1 by construction

$$
\sum_{j=1}^{m} \alpha_{j}(\mathbf{x}, W) \nabla f_{j}(\mathbf{x}, W)\left(\mathbf{x}-\mathbf{x}^{\perp}\right) \geq \frac{C}{2}\left\|\mathbf{x}-\mathbf{x}^{\perp}\right\|^{2} \text { a.s.. }
$$

The proof follows by taking the expectation

$$
\Xi^{\star}(\mathbf{x})\left(\mathbf{x}-\mathbf{x}^{\perp}\right)=\sum_{j=1}^{m} \mathbb{E}\left[\alpha_{j}(\mathbf{x}, W) \nabla f_{j}(\mathbf{x}, W)\right]\left(\mathbf{x}-\mathbf{x}^{\perp}\right) \geq \frac{C}{2}\left\|\mathbf{x}-\mathbf{x}^{\perp}\right\|^{2} .
$$

Remark 4 (Weaker hypothesis H5). The approach of keeping the same hypothesis as the mono-objective does not take into account the Pareto order. This makes the hypothesis very strong, because the relation

$$
f_{j}(\mathbf{x}, W)-f_{j}\left(\mathbf{x}^{\perp}, W\right) \geq \frac{c_{j}}{2}\left\|\mathbf{x}-\mathbf{x}^{\perp}\right\|^{2} \text { a.s. }
$$


is supposed true for all objectives $(j=1, \ldots, m)$. Using the Pareto dominance approach, we can easily weaken this hypothesis. Considering that $\mathbf{x}^{\perp}$ dominates almost surely the point $\mathbf{x}$ and that the inequality of hypothesis $H 5$ is true for at least one objective $(\ell \in \llbracket 1, m \rrbracket)$, it is possible to demonstrate the same property for the mean descent vector

$$
\left\{\begin{array}{l}
\exists \ell \in \llbracket 1, m \rrbracket, f_{\ell}(\mathbf{x}, W)-f_{\ell}(\mathbf{x}, W) \geq \frac{c_{\ell}}{2}\left\|\mathbf{x}-\mathbf{x}^{\perp}\right\|^{2} \\
\forall j \in \llbracket 1, m \rrbracket \backslash\{\ell\}, f_{j}(\mathbf{x}, W)-f_{j}(\mathbf{x}, W) \geq 0
\end{array}\right.
$$

It follows immediately that

$$
\xi^{\star}(\mathbf{x}, W)\left(\mathbf{x}-\mathbf{x}^{\perp}\right) \geq \alpha_{\ell}(\mathbf{x}, W) \frac{c_{\ell}}{2}\left\|\mathbf{x}-\mathbf{x}^{\perp}\right\|^{2} \text { a.s., }
$$

and therefore,

$$
\Xi^{\star}(\mathbf{x})\left(\mathbf{x}-\mathbf{x}^{\perp}\right) \geq \mathbb{E}\left[\alpha_{\ell}(\mathbf{x}, W)\right] \frac{c_{\ell}}{2}\left\|\mathbf{x}-\mathbf{x}^{\perp}\right\|^{2}
$$

\subsubsection{Mean square convergence of SMGDA}

We introduce the filtration $\left(\mathcal{F}_{k}\right)_{k \in \mathbb{N}}$ where the $\sigma$-algebras are generated by the $k$ first random variables of the sequence $\left(W_{n}\right)_{n \in \mathbb{R}^{n}}$

$$
\mathcal{F}_{k}=\sigma\left(W_{1}, \ldots, W_{k}\right)
$$

By construction, the random variable $\mathbf{X}_{k}$ is $\mathcal{F}_{k}$-measurable for any $k \in \mathbb{N}$. From now on, we denote the common descent vector $\xi^{\star}\left(\mathbf{X}_{k}, W_{k+1}\right)$ by the notation

$\xi_{k}^{\star}$ and we use $\Xi_{k}^{\star}$ for the mean descent vector $\Xi^{\star}\left(\mathbf{X}_{k}\right)$.

\section{Lemma 4.}

$\mathbb{E}\left[\left\langle\mathbf{X}_{k}-\mathbf{X}_{k}^{\perp}, \xi_{k}^{\star}-\Xi_{k}^{\star}\right\rangle \mid \mathcal{F}_{k}\right]=0$ a.s.

Proof. This results directly from Lemma 3 since the random variable $W_{k+1}$ is independent from the $\sigma$-algebra $\mathcal{F}_{k}$

$$
\mathbb{E}\left[\left\langle\mathbf{X}_{k}-\mathbf{X}_{k}^{\perp}, \xi_{k}^{\star}-\Xi_{k}^{\star}\right\rangle \mid \mathcal{F}_{k}\right]=\varphi\left(\mathbf{X}_{k}\right) \text { a.s. }
$$

with $\varphi$ the function defined by

$$
\forall \mathbf{x} \in \mathbb{R}^{n}, \varphi(\mathbf{x})=\left\langle\mathbf{x}-\mathbf{x}^{\perp}, \mathbb{E}\left[\xi^{\star}\left(\mathbf{x}, W_{k+1}\right)\right]-\Xi^{\star}(\mathbf{x})\right\rangle .
$$


The conclusion follows from the definition of the mean descent vector

$$
\Xi^{\star}(\mathbf{x})=\mathbb{E}_{W_{k+1}}\left[\xi^{\star}\left(\mathbf{x}, W_{k+1}\right)\right]
$$

Theorem 3. The sequence of random variables $\mathbf{X}_{0}, \mathbf{X}_{1}, \ldots, \mathbf{X}_{n}$ constructed us ing the SMGDA algorithm converges in mean square towards a point $\mathbf{X}^{\perp}$ of the Pareto set $\mathcal{P}_{D}^{\star}$

$$
\lim _{k \rightarrow+\infty} \mathbb{E}\left[\left\|\mathbf{X}_{k}-\mathbf{X}_{k}^{\perp}\right\|^{2}\right]=0 .
$$

Proof. Let $\mathcal{L}_{k}^{\perp}$ denote the square distance between $\mathbf{X}_{k}$ and one of its closest point in $\mathcal{P}_{D}^{\star}: \mathbf{X}_{k}^{\perp}$

$$
\mathcal{L}_{k}^{\perp}=\left\|\mathbf{X}_{k}-\mathbf{X}_{k}^{\perp}\right\|^{2}
$$

As $\mathbf{X}_{k+1}^{\perp}$ is one of the closest point of $\mathcal{P}_{D}^{\star}$ to $\mathbf{X}_{k+1}$, we have

$$
\mathcal{L}_{k+1}^{\perp} \leq\left\|\mathbf{X}_{k+1}-\mathbf{X}_{k}^{\perp}\right\|^{2}
$$

We now introduce the recurrence relation which describes the SMGDA algorithm

$$
\forall k \in \mathbb{N}, \mathbf{X}_{k+1}=\mathbf{X}_{k}-\epsilon_{k} \xi_{k}^{\star}
$$

into the latest relation

$$
\begin{aligned}
\mathcal{L}_{k+1}^{\perp} & \leq\left\|\mathbf{X}_{k}-\epsilon_{k} \xi_{k}^{\star}-\mathbf{X}_{k}^{\perp}\right\|^{2} \\
& \leq \mathcal{L}_{k}^{\perp}+\epsilon_{k}^{2}\left\|\xi_{k}^{\star}\right\|^{2}-2 \epsilon_{k}\left\langle\mathbf{X}_{k}-\mathbf{X}_{k}^{\perp}, \xi_{k}^{\star}\right\rangle
\end{aligned}
$$

Adding the null term

$$
\left\langle\mathbf{X}_{k}-\mathbf{X}_{k}^{\perp}, \Xi_{k}^{\star}-\Xi_{k}^{\star}\right\rangle
$$

to the right hand side of the last relation

$$
\mathcal{L}_{k+1}^{\perp} \leq \mathcal{L}_{k}^{\perp}+\epsilon_{k}^{2}\left\|\xi_{k}^{\star}\right\|^{2}-2 \epsilon_{k}\left\langle\mathbf{X}_{k}-\mathbf{X}_{k}^{\perp}, \xi_{k}^{\star}-\Xi_{k}^{\star}+\Xi_{k}^{\star}\right\rangle
$$

Using the results of Propositions 1 and 2, we obtain

$$
\mathcal{L}_{k+1}^{\perp} \leq \mathcal{L}_{k}^{\perp}\left(1-\epsilon_{k} C\right)+\epsilon_{k}^{2} M_{\xi^{\star}}^{2}-2 \epsilon_{k}\left\langle\mathbf{X}_{k}-\mathbf{X}_{k}^{\perp}, \xi_{k}^{\star}-\Xi_{k}^{\star}\right\rangle
$$


We then take the conditional expectation of the expression with respect to the element $\mathcal{F}_{k}$ of the filtration $(\mathcal{F})$

$$
\mathbb{E}\left[\mathcal{L}_{k+1}^{\perp} \mid \mathcal{F}_{k}\right] \leq \mathbb{E}\left[\mathcal{L}_{k}^{\perp}\left(1-\epsilon_{k} C\right)+\epsilon_{k}^{2} M_{\xi^{\star}}^{2}-2 \epsilon_{k}\left\langle\mathbf{X}_{k}-\mathbf{X}_{k}^{\perp}, \xi_{k}^{\star}-\Xi_{k}^{\star}\right\rangle \mid \mathcal{F}_{k}\right]
$$

Since the random variable $\mathbf{X}_{k}$ is $\mathcal{F}_{k}$-measurable, we can write

$$
\mathbb{E}\left[\mathcal{L}_{k+1}^{\perp} \mid \mathcal{F}_{k}\right] \leq \mathcal{L}_{k}^{\perp}\left(1-\epsilon_{k} C\right)+\epsilon_{k}^{2} M_{\xi}^{2}-2 \epsilon_{k} \mathbb{E}\left[\left\langle\mathbf{X}_{k}-\mathbf{X}_{k}^{\perp}, \xi_{k}^{\star}-\Xi_{k}^{\star}\right\rangle \mid \mathcal{F}_{k}\right]
$$

Introducing the result of Lemma 4 yields the following relation

$$
\mathbb{E}\left[\mathcal{L}_{k+1}^{\perp} \mid \mathcal{F}_{k}\right] \leq \mathcal{L}_{k}^{\perp}\left(1-\epsilon_{k} C\right)+\epsilon_{k}^{2} M_{\xi^{\star}}^{2}
$$

Since $\mathbb{E}\left[\mathbb{E}\left[\mathcal{L}_{k+1}^{\perp} \mid \mathcal{F}_{k}\right]\right]=\mathbb{E}\left[\mathcal{L}_{k+1}^{\perp}\right]$

$$
\mathbb{E}\left[\mathcal{L}_{k+1}^{\perp}\right] \leq \mathbb{E}\left[\mathcal{L}_{k}^{\perp}\right]\left(1-\epsilon_{k} C\right)+\epsilon_{k}^{2} M_{\xi^{\star}}^{2} .
$$

Considering the above relation for $N$ consecutive terms, we have

$$
\mathbb{E}\left[\mathcal{L}_{k+1+N}^{\perp}\right] \leq \mathbb{E}\left[\mathcal{L}_{k}^{\perp}\right] \prod_{\ell=k}^{k+N}\left(1-C \epsilon_{\ell}\right)+\sum_{\ell=k}^{k+N} \epsilon_{\ell}^{2} M_{\xi^{\star}}^{2} .
$$

The proof of the convergence follows from the fact that the two sequences $\sum_{\ell=k}^{k+N} \epsilon_{\ell}^{2} M_{\xi^{\star}}^{2}$ and $\prod_{\ell=k}^{k+N}\left(1-C \epsilon_{\ell}\right)$ converge towards 0 , the first one because $\left(\epsilon_{k}\right)_{k \in \mathbb{N}}$ is a $\sigma$-sequence, the second one because of the convergence of its logarithm image. Finally we have proved that

$$
\lim _{N \rightarrow \infty}\left(\mathbb{E}\left[\mathcal{L}_{k+1+N}^{\perp}\right]\right)=0
$$

185

which proyes the mean square convergence theorem.

A convergence speed result is also available for the mean square convergence.

Theorem 4. Let $\mathbf{X}_{0}$ be an initial design point for the stochastic optimization problem. If the following $\sigma$-sequence is used in the algorithm

$$
\epsilon_{k}=\frac{1}{k C / 2+\frac{M_{\xi}^{2}}{\mathbb{E}\left[\mathcal{L}_{0}^{\perp}\right] C / 2}}
$$


then

$$
\mathbb{E}\left[\mathcal{L}_{k}^{\perp}\right] \leq \frac{1}{\frac{(C / 2)^{2}}{M_{\xi}^{2}} k+\frac{1}{\mathbb{E}\left[\mathcal{L}_{0}^{\perp}\right]}}
$$

The proof of this convergence speed is exactly the same as the one given in [19] for mono-objective problems and will not be recalled here.

Remark 5. It can be seen that the convergence speed depends on the chosen $\sigma$-sequence.

\subsubsection{Almost sure convergence of SMGDA}

Theorem 5. The sequence of random variables $\mathbf{X}_{0}, \mathbf{X}_{1}, \ldots, \mathbf{X}_{n}$ constructed using the SMGDA algorithm converges almost surely towards a point $\mathbf{X}^{\perp}$ of the Pareto set $\mathcal{P}_{D}^{\star}$

$$
\mathbb{P}\left(\left\{\lim _{k \rightarrow \infty}\left(\mathbf{X}_{k}-\mathbf{X}_{k}^{\perp}\right)=0\right\}\right)=1 .
$$

Proof. Let $\left(Y_{k}\right)_{k \in \mathbb{N}}$ be the random sequence defined by

$$
Y_{k}=\left\|\mathbf{X}_{k}-\mathbf{X}_{k}^{\perp}\right\|^{2}+M_{\xi^{\star}}^{2} \sum_{\ell \geq k} \epsilon_{\ell}^{2} .
$$

Using the inequality (29), we can write

$$
Y_{k+1} \leq\left\|\mathbf{X}_{k}-\mathbf{X}_{k}^{\perp}\right\|^{2}+\epsilon_{k}^{2} M_{\xi^{\star}}^{2}+\sum_{\ell \geq k+1} \epsilon_{\ell}^{2} M_{\xi^{\star}}^{2}-2 \epsilon_{k}\left\langle\mathbf{X}_{k}-\mathbf{X}_{k}^{\perp}, \xi_{k}^{\star}-\Xi_{k}^{\star}+\Xi_{k}^{\star}\right\rangle .
$$

Proposition 2 allows us to bound from above by 0 the last term of this relation

$$
-2 \epsilon_{k}\left\langle\mathbf{X}_{k}-\mathbf{X}_{k}^{\perp}, \Xi_{k}^{\star}\right\rangle \leq-\epsilon_{k} C\left\|\mathbf{X}_{k}-\mathbf{X}_{k}^{\perp}\right\|^{2} \leq 0
$$

which leads to the following inequation

$$
Y_{k+1} \leq\left\|\mathbf{X}_{k}-\mathbf{X}_{k}^{\perp}\right\|^{2}+\epsilon_{k}^{2} M_{\xi^{\star}}^{2}+\sum_{\ell \geq k+1} \epsilon_{\ell}^{2} M_{\xi^{\star}}^{2}-2 \epsilon_{k}\left\langle\mathbf{X}_{k}-\mathbf{X}_{k}^{\perp}, \xi_{k}^{\star}-\Xi_{k}^{\star}\right\rangle
$$

Taking the conditional expectation of $Y_{k+1}$ with respect to the $\sigma$-algebra $\mathcal{F}_{k}$, we obtain

$$
\mathbb{E}\left[Y_{k+1} \mid \mathcal{F}_{k}\right] \leq \mathbb{E}\left[Y_{k} \mid \mathcal{F}_{k}\right]-2 \epsilon_{k} \mathbb{E}\left[\left\langle\mathbf{X}_{k}-\mathbf{X}_{k}^{\perp}, \xi_{k}^{\star}-\Xi_{k}^{\star}\right\rangle \mid \mathcal{F}_{k}\right]
$$


Knowing that $Y_{k}$ is $\mathcal{F}_{k}$-mesurable and using the Lemma 4 we finally obtain the following expression

$$
\mathbb{E}\left[Y_{k+1} \mid \mathcal{F}_{k}\right] \leq Y_{k}
$$

The random process $\left(Y_{k}\right)_{k \in \mathbb{N}}$ is a supermartingale which is obviously positive. Therefore, using Theorem 2, the random process $\left(Y_{k}\right)_{k \in \mathbb{N}}$ converges almost surely toward a random variable $Y_{\infty}$. Using Fatou's lemma, we can now bound the random variable $Y_{\infty}$ by the following expression

$$
\begin{aligned}
0 \leq \mathbb{E}\left[\lim _{k \rightarrow \infty}\left(\inf _{\ell \geq k} Y_{\ell}\right)\right]=\mathbb{E}\left[Y_{\infty}\right] & \leq \lim _{k \rightarrow \infty}\left(\inf _{\ell \geq k} \mathbb{E}\left[Y_{\ell}\right]\right) \\
& \leq \lim _{k \rightarrow \infty}\left(\mathbb{E}\left[\mathcal{L}_{k}^{\perp}\right]+\sum_{\ell \geq k} \epsilon_{\ell}^{2} M_{\xi^{\star}}^{2}\right)
\end{aligned}
$$

The mean square convergence and the fact that the second term is the remainder of the $2^{n d}$ order series of $\left(\epsilon_{k}\right)$ allow us to deduce that

$$
\mathbb{E}\left[Y_{\infty}\right]=0
$$

Knowing that $\left(Y_{k}\right)$ is a positive random process implies that $Y_{\infty}=0$ almost surely

\section{Illustrations}

$$
\mathbb{P}\left(\lim _{k \rightarrow \infty} Y_{k}=0\right)=1 \text {. }
$$

The efficiency and the reliability of the method is assessed by comparing the solution obtained by $S M G D A$ and by two other solvers : NSGA-II [10], and $D M S[8]$ on several classical deterministic benchmark problems described in [27]. The problems are chosen in order to present different situations : convex, nonconvex and discontinuous Pareto sets. Uncertainties are added to each problem by introducing random variables into the objective functions. Since the expectations appearing in the optimization problem described by equation 
(24) are not available, a sample average approximation approach is used for NSGA-II and DMS in order to evaluate them:

$$
\mathbb{E}[f(\mathbf{x}, W)] \approx \frac{1}{N} \sum_{i=1}^{N} f\left(\mathbf{x}, w_{i}\right)
$$

where $w_{i}$ are independent samples of the random variable $W$. The number $N$ of samples plays a crucial role in the efficiency of the algorithm: a too small value results in a wide confidence interval and a poor estimate of the objective function, while an excessive value results in a dramatic increase of the computational cost. The test cases are conducted taking into account a budget based on the maximum number of calls to the objective functions. In order to compare the performance of the three algorithms tested in this section, two classical indicators are introduced: a performance indicator called Purity [3], which compares the number of non-dominated points an algorithm is able to find to a reference front built using the results of the three optimizers, and the well known Hypervolume indicator [2] which gives an indication on both the spreading of the front and its quality by calculating the sum of the hypervolumes generated between all non-dominated points and a reference point taken in the objective space. This last metric is illustrated in Figure 4, where the reference point is represented by the symbol and where the hypervolume corresponds to the green area. For both indicators the higher the score is the better the performance is. The tuning of NSGA-II and DMS parameters (including the number of samples used for the sample average approximation) is not straightforward and we used an auxiliary genetic algorithm in order to find the parameter values which maximizes the resulting Hypervolume measure for each problem. Due to the stochastic nature of the problems the fitness considered is the mean value of the resulting Hypervolume measure estimated on a sample of 5 independent runs for the same set of parameter values. The tuning optimizer is run for a population of 20 individuals and 15 generations.

In this section we shall begin by presenting in details the $M O P 2$ test case : the exact formulation of the problem is written, the insertion of the uncertainties and the results of each algorithm are detailed. The results of the other 
test cases are analyzed using performance profiles which gives an indication of performance on the overall set of problems [20].

\subsection{MOP2 problem}

The first test case presented is a randomized version of the MOP2 test case [27]. It involves two non convex objective functions and fifteen design variables:

$$
\min _{\mathbf{x} \in[-4,4]^{15}}\left\{\begin{array}{l}
\mathbb{E}\left[f_{1}(\mathbf{x}, W)\right]=\mathbb{E}\left[1-\exp \left(-\sum_{i=1}^{15}\left(\mathbf{x}_{i}-\frac{1+W_{1 i}}{\sqrt{15}}\right)^{2}\right)\right] \\
\mathbb{E}\left[f_{2}(\mathbf{x}, W)\right]=\mathbb{E}\left[1-\exp \left(-\sum_{i=1}^{15}\left(\mathbf{x}_{i}+\frac{1+W_{2}}{\sqrt{15}}\right)^{2}\right)\right]
\end{array}\right\}
$$

225 where $\left\{W_{1 i}\right\}_{i \in\{1,15\}}$ and $\left\{W_{2 i}\right\}_{i \in\{1,15\}}$ are thirty independent random variables with uniform distribution on the interval $[-0.7,0.7]$. We shall denote $W$ the random vector $\left(W_{1, i} ; W_{2, i}\right) i \in\{1,15\}$.

The reference point considered for defining the Hypervolume indicator is the point $f_{\text {ref }}=(1.1,1.1)$. As it is explained above, $N S G A-I I$ and DMS parameters have been optimized using a genetic algorithm in order to maximize the Hypervolume measure calculated with $f_{\text {ref }}$ as the reference point. Ten independent runs of NSGA-II and DMS with the optimized parameters are generated. The run with the highest hypervolume is the only one considered in the results. A budget of $10^{5}$ calls to the objective functions is allocated for both NSGAII and DMS algorithms while only $10^{4}$ calls are allocated to SMGDA and they are shared over $10^{2}$ initial points. Once the three algorithms are stopped, a last estimation of the final mean performance is done using a sample average approximation with $10^{5}$ samples. For this particular problem, NSGA-II parameters are set to a population of 211 individuals over 236 generations. The crossover probability is tuned to $92 \%$, and the crossover index to 1.05 . The mutation index parameter has shown to have a very low influence on the Hypervolume measure result. Thus it has not been considered in the parameter optimization and was set to 10 in all problems. The sample average approximation uses 2 samples which is very small, but it gives the best Hypervolume measure for the $10^{5}$ allowed calls. The NSGA-II results are expected to have a low Purity score as accuracy will be impacted by the small number of samples used for 
the expectation approximations. The number of samples for $D M S$ was set to 45 which allows to use more than 2000 calls to the objective functions. No step size criterion was imposed for $D M S$ algorithm. The critical tuning of $S M G D A$ lies in its $\sigma$-sequence that rules the step size. For the MOP2 problem,we used the sequence

$$
\epsilon_{k}=\frac{10^{-1}}{k+1}
$$

$k$ being the algorithm iteration index.

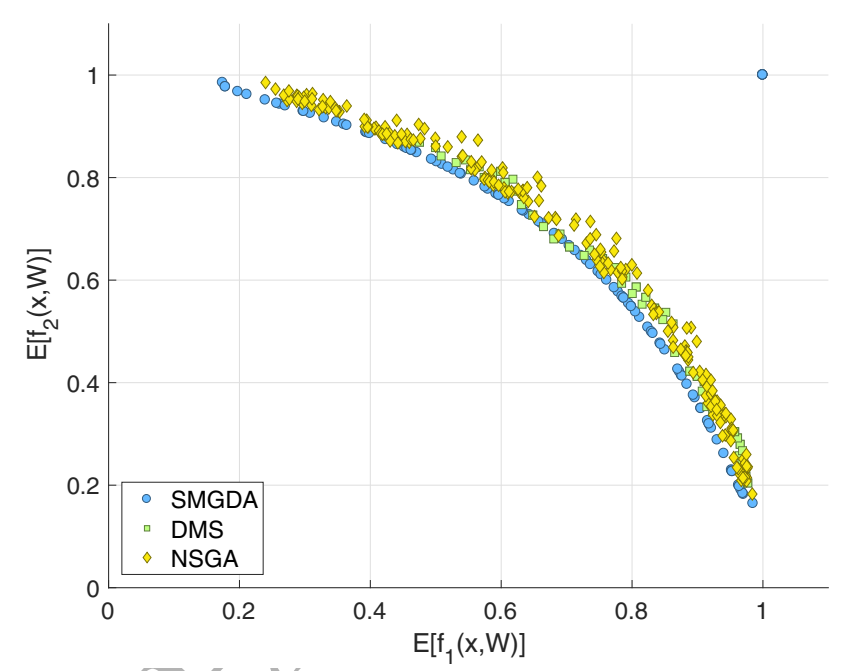

Figure 1: Pareto fronts given by the three solvers for the $M O P 2$ problem

Looking at the Pareto front found by the three algorithm and illustrated on ${ }_{230}$ Figure 1, SMGDA seems to give the best results: most of the solutions found by $S M G D A$ are dominating the solutions provided by the other solvers. This is confirmed by looking at the Purity metric illustrated in Figure 2a. Due to the gradient low values of the objective functions far from the front, some points of $S M G D A$ have not been able to converge for the allowed budget. Analyzing 235 the Hypervolume metric results for this particular problem, $S M G D A$ is able to give results very close to the maximum obtained by combining the solutions of the three algorithms together which define the reference Pareto front used for 
characterizing the Purity metric.

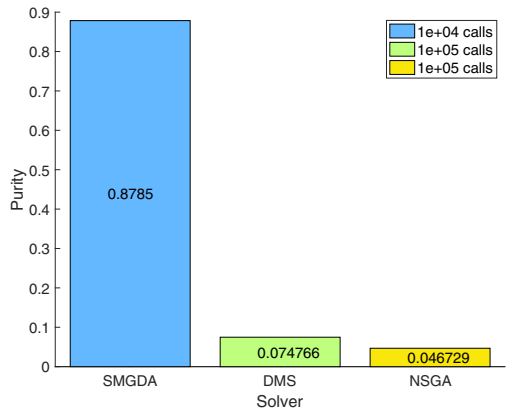

(a) Purity

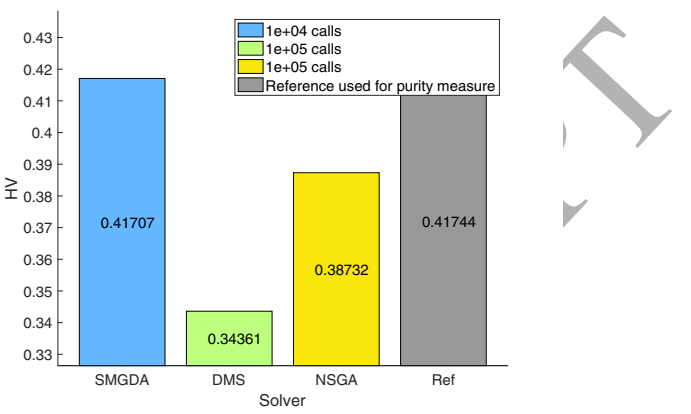

(b) Hypervolume measure

Figure 2: Performance indicators for MOP2 problem

In order to illustrate the impact of uncertainties on the solutions, the probability distribution of the $S M G D A$ solutions was also built. For each converged design point $\mathrm{x}^{*}$ in the Pareto set, $10^{5}$ independent samples of the random functions $f_{i}\left(\mathbf{x}^{*}, W\right)$ have been generated, each sample yielding a Pareto front, solution of a new optimization problem. A Gaussian kernel density estimator was then used in order to build the probability distribution of the random Pareto front. It can be seen on Figure 3 that this distribution has two peaks located at each edge of the front and that it is rather widely spread out in the middle. This indicates that the uncertain parameters have a much greater impact on the solution of the multiobjective optimization problem than on each single objective optimization problem.

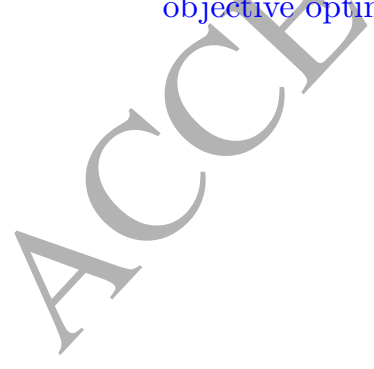




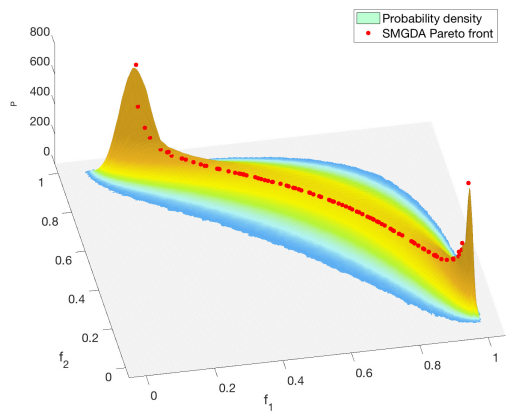

(a) Side view

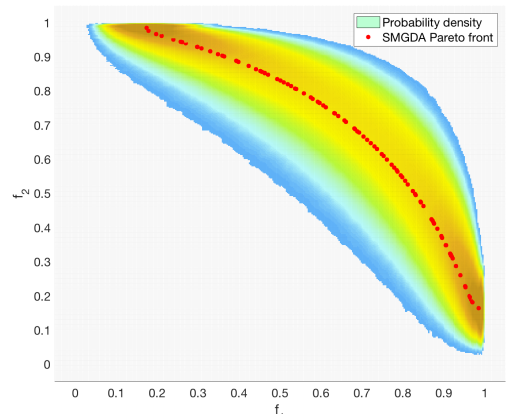

(b) Top view

Figure 3: Density of probability of the Pareto front given by the $S M G D A$ algorithm

\subsection{Additional numerical tests}

The three algorithms are now compared using several other benchmark tests described in in table 1 . The last two columns give the number of total calls budget during the optimization process for each algorithm tested. The number of calls allowed for SMGDA is set to be 10 times less than for DMS and NSGA-II .

In this section the Pareto front is not drawn for each problem, but performance profiles using Purity and Hypervolume metrics are used in order to compare the performance of the three algorithms. The performance profiles correspond to a cumulative distribution function that gives an indication of the percentage of problems considered solved for a certain threshold $\tau$ of the ratio

$$
r_{p, s}=\frac{t_{p, s}}{\min \left\{t_{p, \bar{s}}, \bar{s} \in \mathcal{S}\right\}}
$$

where $p$ is an optimization problem belonging to the set $\mathcal{P}$ of benchmark problems addressed, $s$ is the solver used in $\mathcal{S}=\{S M G D A, D M S, N S G A-I I\}$ and $t$ is a performance indicator for which a lower score indicates a better performance. Thus, in this section, it is actually the inverse of Purity and Hypervolume metrics which are used. This leads, for each solver, to the following expression of 


\begin{tabular}{|l|l|l|l|l|}
\hline Problem & $\begin{array}{l}\text { design } \\
\text { variable } \\
\text { number }\end{array}$ & $\begin{array}{l}\text { random } \\
\text { variable } \\
\text { number }\end{array}$ & SMGDA calls & $\begin{array}{l}\text { NSGA-II \& } \\
D M S \text { calls }\end{array}$ \\
\hline MOP2 & 15 & 30 & $10^{4}$ & $10^{5}$ \\
\hline MOP3 & 2 & 18 & $10^{3}$ & $10^{4}$ \\
\hline MOP6 & 2 & 3 & $10^{3}$ & $10^{4}$ \\
\hline ZDT $\{1,2,3\}$ & 30 & 32 & $5.10^{3}$ & $5.10^{4}$ \\
\hline JOS1 & 30 & 60 & $5.10^{3}$ & $5.10^{4}$ \\
\hline JOS2 & 30 & 32 & $5.10^{3}$ & $5.10^{4}$ \\
\hline SCH1 & 1 & 4 & $10^{3}$ & $10^{4}$ \\
\hline IM1 & 2 & 3 & $10^{3}$ & $10^{4}$ \\
\hline
\end{tabular}

Table 1: Benchmark problems

the cumulative distribution function $\rho(\tau)$

$$
\rho_{s}(\tau)=\frac{1}{|\mathcal{P}|} \times\left|\left\{p \in \mathcal{P}, r_{p, s} \leq \tau\right\}\right| .
$$

Thus, for each solver $s$, the value of $\rho_{s}(1)$ is the number of problems for which the performance of algorithm $s$ is superior to the other two.

The set of problems studied covers a large range of optimization problems with convex and non convex objective functions. Two problems with a multimodal objective function are considered in this benchmark (MOP6, ZDT3). Because these two last problems have Pareto stationary points which are not Pareto optimal, SMGDA converges to a Pareto optimal point only when the initialization permits to avoid local minima. This can be observed on Figure 4 and shows that $S M G D A$ should be used with care when dealing with multimodal objectives.

For $\tau$ equal to 1, SMGDA outperforms the other two algorithms. Regarding the performance profiles based on the Purity metric represented on Figure 5, $S M G D A$ has a better performance for eight test problems and is the only algorithm able to reach $\rho_{s}=1$. Which means that $N S G A-I I$ and DMS have not 


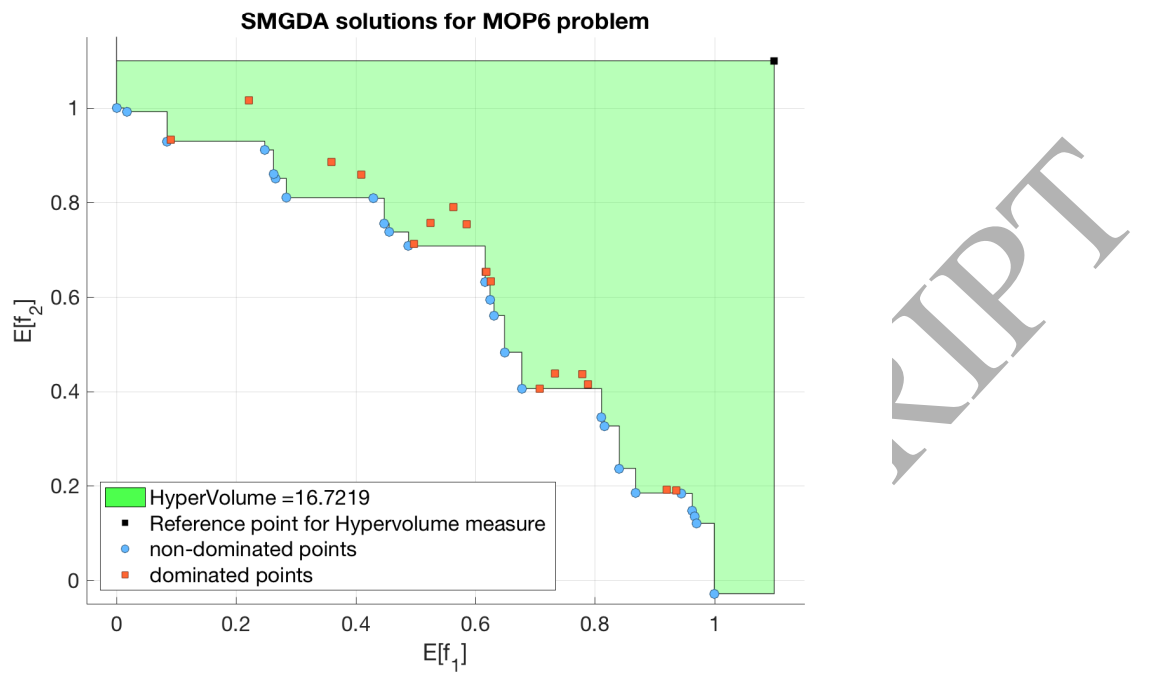

Figure 4: SMGDA solutions for $M O P 6$ problem and its Hypervolume measure problems addressed.

Since the parameters of both DMS and NSGA-II have been optimized specifically for the Hypervolume metric, the two algorithms show a better performance profile than the ones constructed for the Purity metric as it is illustrated in Figure 6. NSGA-II algorithm, especially, outperforms the other two algorithms for

been able generate any non-dominated point on some of the addressed problems for the allocated budget. Because SMGDA may converge towards Pareto stationary but not optimal points, SMGDA obtains a good score using the $\mathrm{Pu}$ rity metric for all the problems except for the two multimodal test cases. But the algorithm reach nevertheless the value of $\rho_{s}=1$ for $\tau=2$ which means that the performance of $S M G D A$ in terms of Purity measure was found inferior to the best performance by a factor of 2 only. Whereas, for certain test cases, DMS (resp. NSGA-II ) can result in a Purity score up to 10 (resp. 100) times lower than the winning algorithm. This demonstrates the capability of $S M G D A$ to perform well and to give good quality results for all the benchmark three test problems. Even if the SMGDA performance is lower for the Hypervolume metric, it is nevertheless rather efficient since the performance reaches 


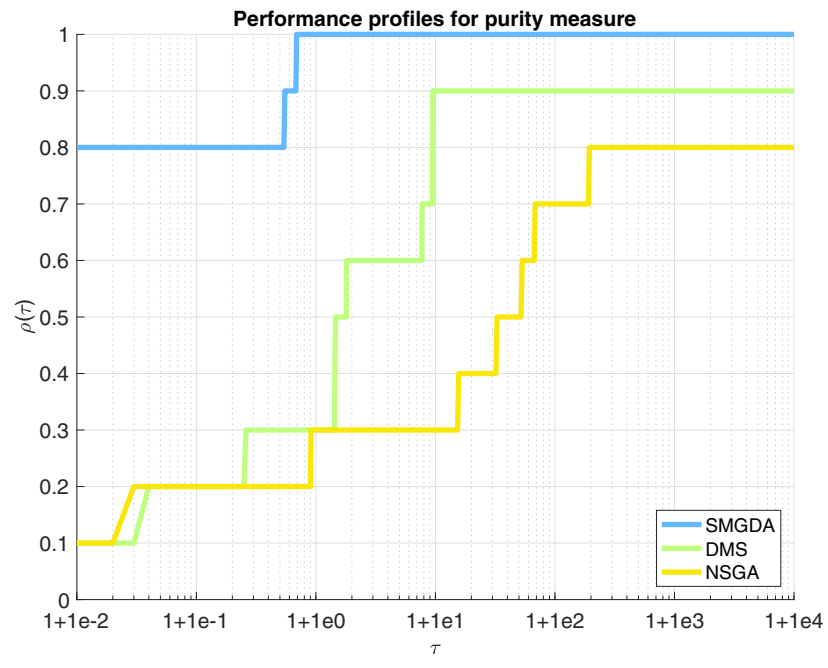

P

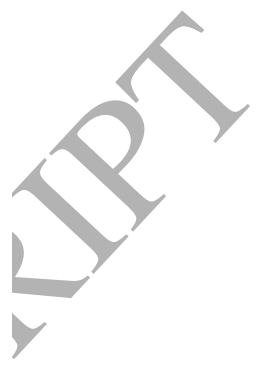

Figure 5: Performance profile of the Purity indicator

$\rho_{s}=1$ for a very low value of $\tau$. For its less performing test case, SMGDA performance score was only 1.031 times less than the best algorithm performance.

The performance profiles presented in this section show that SMGDA can compete successfully with/lassical algorithms used for multiobjective optimization problems, at least for the two performance metric introduced. The numerical efficiency of SMGDA comes mainly from the fact that no estimator construction is necessary to evaluate the objective functions. Moreover the algorithm efficiency does not depend on the number of random variables introduced in the objective functions nor on the number of objective functions. The weakness of the method is the necessity to have the gradient analytic expressions, their

numerical calculation would of course increase the computation time.

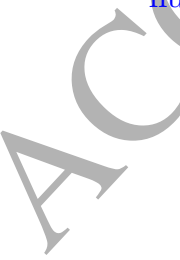




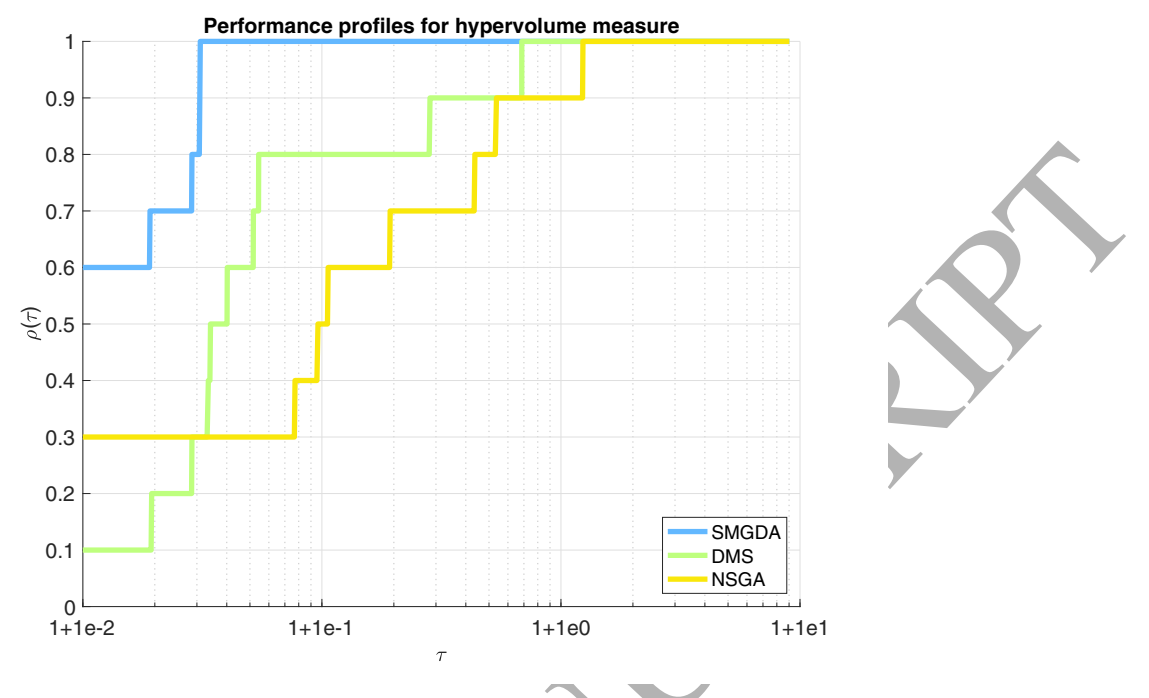

Figure 6: Performance profile of the Hypervolume indicator

\section{Conclusions}

In this article, we have proposed a novel algorithm for solving a stochastic multiobjective optimization problem. It is based on two ingredients: a common random descent vector and an extension of the classical stochastic gradient algorithm. Because the algorithm necessitates only a single iteration loop, it is less time demanding than classical approaches based on sample averaging approximation methods. Two types of convergence have been proved based on rather restrictive assumptions. As it is the case for stochastic gradient algorithms, no efficient stopping criterion exists. Comparisons with NSGA-II and $D M S$ on a set of benchmark problems have shown the very good behaviour of the proposed method which requires much less iterations to converge than the two other solvers tested. Compared to a genetic algorithm there is no exchange of information between the initial points, which may a priori seem to yield a suboptimal decision but which renders the algorithm entirely and readily parallelizable: the computation time can be divided by the number of threads. Of course the objective functions needs to be regular but the approach should be 
easily extended to nonregular objective functions considering descent direction

obtained by subgradients. We are actually working along this path.

\section{References}

[1] Arnaud, R., Poirion, F. (2014). Optimization of an uncertain aeroelastic system using stochastic gradient approaches. Journal of Aircraft, 51(3), 1061-1066.

${ }_{320}$ [2] Auger, A., Bader, J., Brockhoff, D., Zitzler, E. (2009). Theory of hyervolume indicator: optimal $\mu$-distributions and the choice of the reference point. Proceeding of the Tenth ACM SIGEVO Workshop on Foundations of Genetic Algorithm, 87-102.

[3] Bandyopadhyay, S., Pal, S. K., Aruna, B. (2004). Multiobjective GAs, quantitive indices and pattern classification. IEEE Transaction on Systems, Man, and Cybernetics - Part B: Cybernetics, 34(5), 2088-2099.

[4] Bellman, R. E., Zadeh, L. A. (1970). Decision-making in a fuzzy environment. Management Science, 17(4), B-141-B-164.

[5] Bonnel, H., Collonge, J. (2014). Stochastic optimization over a pareto set associated with a stochastic multi-objective optimization problem. Journal of Optimization Theory and Applications, 162(2), 405-427.

[6] Boyd, S.) Vandenberghe, L. (2004). Convex Optimization. Cambridge: Cambridge University Press.

[7] Cabạllero, R., Cerdá, E., del Mar Muñoz, M., Rey, L. (2004). Stochastic approach versus multiobjective approach for obtaining efficient solutions in stochastic multiobjective programming problems. European Journal of Operational Research, 158(3), 633 - 648.

[8] Custodió, A. L., Madeira, J. F. A., Vaz, A. I. F., Vincente, L. N. (2011). Direct Multisearch for Multobjective Optimization. SIAM Journal on Optimization, 21(3), 1109-1140. 
[9] Dantzig, G. B. (2004). Linear programming under uncertainty. Management Science, 50(12_supplement),1764-1769.

[10] Deb, K., Pratap, A., Agarwal, S., Mayarivan, T. (2002). A fast and elitist multiobjective genetic algorithm: NSGA-II. IEEE Transaction on Evolutionary Computation, 6(2), 181-197.

[11] Deb, K., Gupta, H. (2005). Searching for Robust Pareto-Optimal Solutions in Multi-objective Optimization. Evolutionary Multi-Criterion Optimization. EMO 2005, 3410, 150-164.

[12] Désidéri, J.-A. (2012). Multiple-gradient descent algorithm (MGDA) for multiobjective optimization. Comptes Rendus Mathematique, 3810(5), 229332.

[13] Désidéri, J.-A. (2009). Multiple-gradient descent algorithm (MGDA) (Tech. Rep. No. 6953). Sophia Antipolis: Inria.

[14] Désidéri, J.-A. (2014). Multiple-Gradient Descent Algorithm (MGDA) for Pareto-Front Identification. In W. Fitzgibbon, W. Kuznetsov, Y. Neittaanmäki, O. Pironneau (Eds.), Modeling, Simulation and Optimization for Science and Tchnology (pp. 41-58). Dordrecht, Netherlands: Springer,Dordrecht.

[15] Désidéri, J.-A. (2015). Révision de l'algorithme de descente à gradients multiples (MGDA) par orthogonalisation hiérarchique (Tech. Rep. No. 8710). Sophia Antipolis: Inria.

[16] Désidéri, J.-A. (2018). Quasi-Riemannian Multiple Gradient Descent Algorithm for constrained multiobjective differential optimization (Tech. Rep. No. 9159). Sophia Antipolis: Inria.

365 [17] Désidéri, J.-A., Duvigneau, R. (to appear). Parametric optimization of pulsating jets in unsteady flow by multiple-gradient descent algorithm (MGDA). In B. N. Chetverushkin, W. Fitzgibbon, Y. A. Kuznetsov, P. 
Neittaanmäki, J. Periaux, O. Pironneau (Eds.), Contributions to Partial Differential Equations and Applications, Computational Methods in Applied Sciences. https://www.springer.com/us/book/9783319783246.

[18] Diaz, J. E., Handl, J., Xu, D.-L. (2017). Evolutionary robust optimization in production planning : interactions between number of objectives, sample size and choice of robustness measure. Computers $\&$ Operations Research, $79,266-278$.

[19] Dodu, J. C., Goursat, M., Hertz, A., Quadrat, J.-P., Viot, M.' (1981). Méthodes de gradient stochastique pour l'optimisation des investissements dans un réseau électrique. EDF, Bulletin de la Direction des études et recherches, Série C, Mathématique, Informatique, 2, 133-167.

[20] Dolan, E. D., Moré, J. J. (2002). Benchmarking optimization software with performance profiles. Mathematical Programming, 91(2), 201-213.

[21] Ermoliev, Y., Wets, R. J.-B. (1988). Numerical Techniques for Stochastic Optimization. NY: Springer-Verlag New York.

[22] Ermoliev, Y. M., Gaivoronski, A. A. (1992). Stochastic quasigradient methods for optimization of discrete event systems. Annals of Operations Research, 39(1), 1-39.

[23] Fliege, J., Svaiter, B. F. (2000). Steepest descent methods for multicriteria optimization. Mathematical Methods of Operations Research, 51(3), 479494

[24] Fliege, J., Xu, H. (2011). Stochastic multiobjective optimization: Sample average approximation and applications. Journal of Optimization Theory and Applications, 151(1), 135-162.

[25] Fliege, J., Werner, R. (2014). Robust multiobjective optimization \& applications in portfolio optimization. European Journal of Operational Research, 234(2), $422-433$. 
[26] Gabrel, V., Murat, C., Thiele, A. (2014). Recent advances in robust optimization: An overview. European Journal of Operational Research, 235(3), $471-483$.

[27] Huband, S., Hingston, P., Barone, L., While, L. (2006). A Review of Multiobjective Test Problems and a Scalable Test Problem Toolkit. IEEE Transaction on Evolutionary Computation, 10(5), 477-506.

[28] Klamroth, K., Köbis, E., Schöbel, A., Tammer, C. (2017). A unified approach to uncertain optimization. European Journal of Operational Research, 260(2), $403-420$.

[29] Löhndorf, N. (2016). An empirical analysis of scenario generation methods for stochastic optimization. European Journal of Operational Research, $255(1), 121-132$.

[30] Matthies, H. G., Brenner, C. E., Bucher, C. G., Soares,C. G. (1997). Uncertainties in probabilistic numerical analysis of structures and solidsstochastic finite elements. Structural Safety, 19(3), $283-336$.

${ }_{410}$ [31] Mattson, C. A., Messac, A. (2005). Pareto frontier based concept selection under uncertainty, with visualization. Optimization and Engineering, 6(1), $85-115$.

[32] Nemirovski, A., Shapiro, A. (2006). Scenario Approximations of Chance Constraints. In G. Calafiore \& F. Dabbene (Eds.), Probabilistic and Randomized Methods for Design under Uncertainty (pp. 3-47). London, UK: Springer, London.

[33] Papadimitriou, C., Katafygiotis, L. S., Au, S.-K. (1997). Effects of structural uncertainties on tmd design: A reliability-based approach. Journal of Structural Control, 4(1), 65-88.

${ }_{420}$ [34] Polyak, B. (1976). Convergence and convergence rate of iterative stochastic algorithms. Automatica i Telemekhanika, 12, 83-94. 
[35] Robbins, H., Monro, S. (1951). A stochastic approximation method. The Annals of Mathematical Statistics, 22(3), 400-407.

[36] Roy, R., Hinduja, S.,Teti, R. (2008). Recent advances in engineering design optimisation: Challenges and future trends. CIRP Annals, 57(2), 697-715.

[37] Sahinidis, N. V. (2004). Optimization under uncertainty: state-of-the-art and opportunities. Computers $\&$ Chemical Engineering, 28(6), 971 - 983.

[38] Schaffer, J. D. (1984). Some experiments in machine learning usingvector evaluated genetic algorithms (artificial intelligence, optimization, adaptation, pattern recognition) (Ph.D. thesis). Vanderbilt University, Nashville, USA.

[39] Shapiro, A. (2003). Monte Carlo sampling approach to stochastic programming. ESAIM: Proceedings, 13, 65-73.

[40] Wang, Z., Guo, J., Zheng, M., Wang, Y. (2015). Uncertain multiobjective traveling salesman problem. European Journal of Operational Research, $241(2), 478-489$.

[41] Zerbinati, A., Désidéri, J.-A., Duvigneau, R. (2011). Comparison between MGDA and PAES for multiobjective optimization (Tech. Rep. No. 7667). Sophia Antipolis: Inria.

${ }_{440}$ [42] Zitzler, E., Deb, K., Thiele, L. (2000). Comparison of multiobjective evolutionary algorithms: Empirical results. Evolutionary Computation, 8(2), $173-195$.

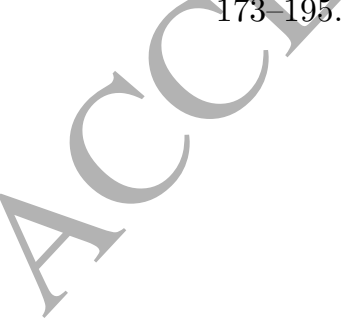

